\title{
A Study on the Effect of J2 Perturbation on Optimum Delta-V for Collision Avoidance Maneuver
}

\author{
Gisa G S \\ Assistant Professor \\ Department of Aerospace Engineering, \\ Alliance University, Bangalore, India
}

\author{
Yadu Krishnan S \\ Assistant Professor \\ Department of Aerospace Engineering, \\ Alliance University, Bangalore, India
}

\author{
Bharath R K \\ Undergraduate student \\ Department of Aerospace Engineering, \\ Alliance University, Bangalore, India
}

\begin{abstract}
The focus of this study aims to understand the probability of collision between any two objects orbiting the earth. Iridium $\mathbf{7 3}$ is considered as the primary satellite and the dead satellite Taurus is considered as the debris for the collision probability study. The numerical results agree favorably with the predicted values from the explicit collision probability method. The collision avoidance maneuver is performed to safeguard the satellite by changing its semi-major axis and bringing back to the same orbit after maneuvering. Considering a range of true anomalies, the optimum delta- $\mathrm{V}$ is obtained by trial and error method. The effect of $\mathrm{J} 2$ perturbation of the earth on the optimum delta- $\mathrm{V}$ is carefully studied and has been observed to be less than that of an unperturbed condition. This study shows the significance of the collision avoidance maneuver and the $\mathrm{J} 2$ perturbation to perform a safe maneuver.
\end{abstract}

Keywords: Collision probability, Collision avoidance maneuver, Optimum delta $V$, J2 perturbation.

\section{INTRODUCTION}

The increase in the population of space comes with a great risk of satellite collision and space pollution. To continue exploring the space, there is a huge task of protecting our space environment. The increasing number of space launches, testing of anti-satellite missile and unexpected collision has led to overcrowding of low earth orbit, which has led to the development of collision probability between primary and secondary satellite. Russell P Patera [1] derives the general formula for calculating collision probability with a single closed path integral in the encounter frame. Patera reduces the threedimensional Gaussian distribution into two-dimensional form by transferring the earth fixed frame into the encounter frame. Then converts the ellipse into a spherical coordinate system and integrates over radius $\mathrm{r}$, which reduces the two-dimensional Gaussian distribution into single integral over ellipse. This equation is valid for all the satellite irrespective of the shape and size. Lie Chen et al. [2] derives the explicit expression for calculating collision probability assuming that the orbits are circular or near-circular orbit. The expression considers only the components of relative position and velocity and their standard deviation of position vectors. The assumption made has circular and near-circular orbit solves the integral from the Patera method thus giving a simplified expression for calculation of collision probability. Xiao-Li Xu et al. [3] develops the formula for calculating collision probability in circular orbit or nearcircular orbit by dividing the collision probability into two separate cases.
The probability of collision due to height difference and probability of collision due to the time of arrival at the encounter frame. Then these two probabilities are multiplied which will give the total collision probability of the satellites. Claudio Bombardelli et al. [4] perform the two and three impulsive burns of the primary satellite (Cosmos) to maneuver the satellite in a collision course with another satellite (Iridium). The maneuver takes earth and satellite as the two-point mass without the effect of any other perturbing forces. The NORAD satellite catalog and CelesTrak [5,6] are the two websites that maintain the orbital parameters of the satellite in a two-line format. This study consists of the calculation of the Collision probability of a satellite using TLE. The study also focuses on the optimization of collision avoidance maneuver of the primary satellite considering earth $\mathrm{J} 2$ perturbation. Numerical analysis is carried out using FreeFlyer software.

\section{METHODOLOGY}

A. Two-line element

The overcrowding of low earth orbit often leads to close flyby or chance of collision of satellites. The CelesTrak SOCRATES maintains the top Close flyby or Conjunction possibility of the satellites at regular intervals. The NORAD satellite catalog or CelesTrak will maintain the Two-line element data. The two-line element data set contains all the required information about the satellite parameter such as its international designators, inclination, and drag, etc. The semimajor axis, which is the only missing Keplerian orbital elements, this can be found using the mean motion. The semimajor axis is given by:

$$
a=\frac{\mu^{1 / 3}}{\left(\frac{2 * n * p i}{86400}\right)^{2 / 3}}
$$

B. Keplerian to cartesian coordinate system

The obtained Keplerian orbital elements had to be converted into the cartesian or perifocal coordinate system, which will convert all the Keplerian orbital elements to position and velocity vector. The combination of position and velocity vectors are referred to as state vectors. This section explains the method to convert orbital elements into state vectors.

The position and velocity vector in Keplerian coordinate system are defined as follows:

$$
r=\frac{h^{2}}{\mu} \frac{1}{1+e * \cos \varnothing} *\left[\begin{array}{c}
\cos \Phi \\
\sin \Phi \\
0
\end{array}\right]
$$




$$
v=\frac{\mu}{h} *\left[\begin{array}{c}
-\sin \Phi \\
e+\cos \Phi \\
0
\end{array}\right]
$$

The final rotation matrix is given by the multiplication of the all the rotation matrix which is given by (Curtis, H. [7])

$Q_{X x}=$

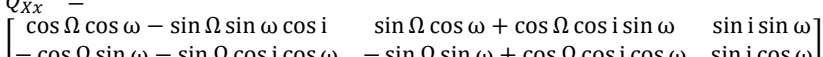
$\left[\begin{array}{ccc}-\cos \Omega \sin \omega-\sin \Omega \cos i \cos \omega & -\sin \Omega \sin \omega+\cos \Omega \cos i \cos \omega & \sin i \cos \omega\end{array}\right]$ The state vectors are obtained by multiplication of position vectors with the rotational matrix.

$$
\begin{array}{r}
\{r\} x=\left[Q_{X x}\right]^{*}[r] \\
\{v\} x=\left[Q_{X x}\right]^{*}[v]
\end{array}
$$

Objects in LEO (low Earth orbit) generally have circular or near circular orbits. The velocity of the satellite at any given point in the circular orbit always remains constant. Since, the velocity and the position vary linearly with time, it is easy to predict the position of the satellite. For a given initial condition, the position at time $t$ is:

$$
X_{1}=X_{0}+V * t
$$

The closest approach is a point where the primary and secondary satellites enter the ellipsoidal range which is determined by differential curve theory. The differential curve theory states that the closest approach between two curves is a point where the position vector is perpendicular to the tangential vector. The relative position of the satellite is determined either by propagating the position vector over time or by STK software which gives directly the relative position and velocity. The close point is the dot product of the relative position and velocity vector. The point where the dot product becomes zero is known as the closest point and time at which the dot product becomes zero is the time of the closest approach.

\section{Calculation of collision probability}

The collision of the satellite is one of the dangerous events that will lead to a cluster of debris that can be harmful to satellites. The collision of the satellite depends on the two parameters. The first parameter is the altitude difference between the satellite and the time of arrival at the collision point. The altitude difference is a radial separation of the satellite and the time of arrival depends on the velocity of the satellite.

$\mathrm{Xu}$ and Xiong, [3] have proposed a method of calculating probability of collision caused by difference in altitude and velocity. The collision probability due to the altitude difference is given by:

$$
P_{r}=\frac{\exp \left(\frac{4\left(\mathrm{r}_{\mathrm{a}}-\Delta \mathrm{h}\right)}{\sqrt{2 \pi} * \sigma \mathrm{r}}\right)}{1+\exp \left(\frac{4\left(\mathrm{r}_{\mathrm{a}}-\Delta \mathrm{h}\right)}{\sqrt{2 \pi} * \sigma \mathrm{r}}\right)}-\frac{\exp \left(\frac{4\left(-\mathrm{r}_{\mathrm{a}}-\Delta \mathrm{h}\right)}{\sqrt{2 \pi} * \sigma \mathrm{r}}\right)}{1+\exp \left(\frac{4\left(-\mathrm{r}_{\mathrm{a}}-\Delta \mathrm{h}\right)}{\sqrt{2 \pi} * \sigma \mathrm{r}}\right)}
$$

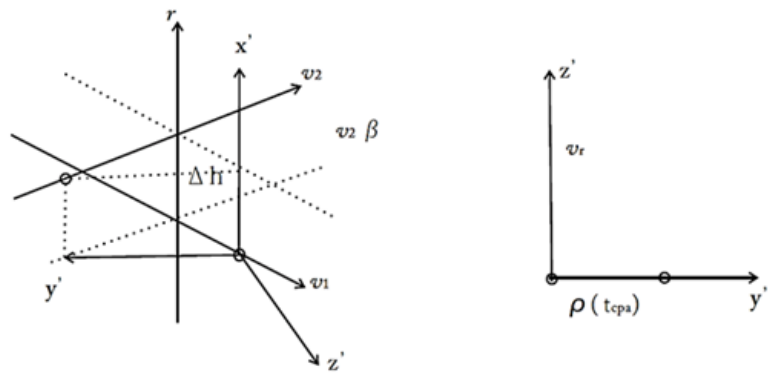

Fig. 1. The encounter frame of primary and secondary satellite [3].

The probability of collision caused by a difference in the arrival time in the cross section is given by:

$$
P_{t}=\frac{\exp \left(\frac{4\left(\mathrm{r}_{\mathrm{t}}-\rho_{\text {min }}\right)}{\sqrt{2 \pi} * \sigma \mathrm{t}}\right)}{1+\exp \left(\frac{4\left(\mathrm{r}_{\mathrm{t}}-\rho_{\text {min }}\right)}{\sqrt{2 \pi} * \sigma \mathrm{t}}\right)}-\frac{\exp \left(\frac{4\left(-\mathrm{r}_{\mathrm{t}}-\rho_{\text {min }}\right)}{\sqrt{2 \pi} * \sigma \mathrm{t}}\right)}{1+\exp \left(\frac{4\left(-\mathrm{r}_{\mathrm{t}}-\rho_{\min }\right)}{\sqrt{2 \pi} * \sigma \mathrm{t}}\right)}
$$

The total collision probability is calculated by multiplying the collision probability along radial and transverse direction:

$$
\mathrm{P}=\mathrm{P}_{\mathrm{r}} * \mathrm{P}_{\mathrm{t}}
$$

Here, $\sigma_{\mathrm{t}}$ and $\sigma_{\mathrm{r}}$ are the relative standard deviation in radial and transverse plane. The $\sigma_{\mathrm{t}}$ and $\sigma_{\mathrm{r}}$ will have to be in the encounter frame in order to calculate collision probability. The error covariance matrix is transferred into the encounter frame [3].

D. J2 Perturbation

Forces that deviate a satellite from a Keplerian orbit are known as perturbations. Examples of perturbations include atmospheric drag, solar radiations, uneven distribution of gravity, third body interaction, and earth magnetic field. The variations of earth mass concentration from pole to equator cause difference in gravitation. The surface of constant gravitation can be more accurately calculated by expressing the geo-potential series as the terms of spherical harmonics. The contribution of any harmonic is determined by a factor represented as $\mathbf{J}$. A large number of $\mathbf{J}$ terms are required to express the gravitational field more accurately, but in practice the higher orders of $\mathbf{J}$ contribute so little to the gravitational variation and it is often neglected. In this study, only the largest perturbation due to $\mathrm{J} 2$ component is considered and its value is \1082.48*10》^(-6) (Bate et al.,[8]).

\section{RESULTS \& DISCUSSIONS}

A. Calculation of collision probability of Taurus and Iridium 73 satellites

The calculation of the collision probability is calculated by considering the Iridium 73 as a primary satellite and Taurus as the secondary satellite. Iridium 73 is US satellite which is launched on May 17, 1998 launched at Air force western test range. Taurus a dead satellite which is in the orbit.

TABLE I: ORBITAL ELEMENTS OF SATELLITES

\begin{tabular}{|c|c|c|}
\hline Parameter & Iridium 73 & Taurus \\
\hline Semi-major axis & 7102.33361 & 7102.33361 \\
\hline Eccentricity & 0.00426 & 0.001848 \\
\hline Inclination & 86.418 & 99.06 \\
\hline RAAN & 223.42 & 330.489 \\
\hline Argument of perigee & 224.119 & 320.512 \\
\hline True anomaly & 216.484 & 127.537 \\
\hline
\end{tabular}


TABLE II: THE STATE VECTORS OF IRIDIUM AND TAURUS SATELLITE ARE AS FOLLOWS

\begin{tabular}{|l|l|l|c|c|c|c|}
\hline Satellite & \multicolumn{1}{|c|}{$\mathbf{X}(\mathbf{k m})$} & $\mathbf{Y}(\mathbf{k m})$ & $\mathbf{Z} \mathbf{( k m})$ & $\mathbf{V}_{\mathbf{x}}(\mathbf{k m} / \mathbf{s})$ & $\mathbf{V}_{\mathbf{y}}(\mathbf{k m} / \mathbf{s})$ & $\mathbf{V}_{\mathbf{z}}(\mathbf{k m} / \mathbf{s})$ \\
\hline Iridium 73 & 260.455063 & -1118.7615 & 7016.9165 & 1.954 & 7.070 & 1.207 \\
\hline Taurus & 100.06813 & -1245.329 & 7016.2507 & -7.556 & -0.259 & 0.250 \\
\hline
\end{tabular}

TABLE III: STANDARD DEVIATION OF THE SATELLITE WHILE PROPAGATING THROUGH TIME

\begin{tabular}{|c|c|c|c|}
\hline Satellite & Standard deviation $\boldsymbol{\sigma}_{\mathbf{x}}$ & Standard deviation $\boldsymbol{\sigma}_{\mathbf{y}}$ & Standard deviation $\boldsymbol{\sigma}_{\mathbf{z}}$ \\
\hline Iridium 73 & 3553.333 & 3556.836 & 5016.92 \\
\hline Taurus & 3596.20 & 3590.97 & 4962.82 \\
\hline
\end{tabular}

The ratio of magnitude of the velocity vector and angle between the velocity vector is:

$$
\begin{gathered}
\eta=\frac{v_{2}}{v_{1}}=\frac{7.565}{7.434}=1.017 \\
\varphi=\cos ^{-1} \frac{v 1 . v 2}{V 1 * V 2}=106.852^{\circ}
\end{gathered}
$$

The proximity zone refers to the area around the satellite which defines the ellipsoidal influence of the satellite. The radial height of $100 \mathrm{~km}$, along track distance of $350 \mathrm{~km}$ and cross track distance of $100 \mathrm{~km}$ is applied with Satellite CG as the origin.

The proximity zone was bound around the satellite of size 100:350:100 km.

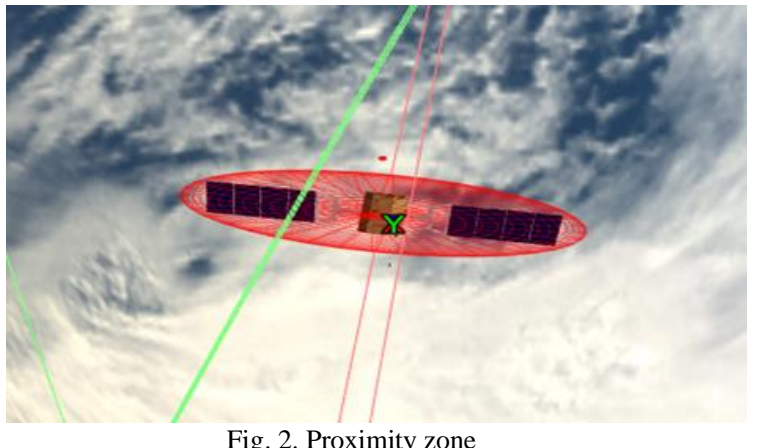

Fig. 2. Proximity zone

The summation of radial distance by considering both the satellite is taken as ra $=100 \mathrm{~km}$ and due to transverse direction is $\mathrm{rt}=350 \mathrm{~km}$. The closest approach distance is obtained by FreeFlyer which is $\rho \min =10817.13 \mathrm{~km}$.

The relative position error in radial direction is:

$$
\sigma_{r}=\sqrt{3553.333^{2}+3596.20^{2}}=5055.5776 \mathrm{~km}
$$

The relative position error in the transverse direction is: $\sigma_{t}^{2}=\frac{\sigma_{1 t}{ }^{2} \eta^{2} \sin \varphi^{2}+(1-\eta \cos \varphi)^{2} \sigma_{1 w}{ }^{2}+\sigma_{2 t}{ }^{2} \sin \varphi^{2}+(\eta-\cos \varphi)^{2} \sigma_{2 w}{ }^{2}}{1+\eta^{2}-2 * \eta * \cos \varphi}==$

$$
=4928.08 \mathrm{~km}
$$

$$
P_{r}=\frac{\exp \left(\frac{4(100-0)}{\sqrt{2 \pi} * 5055.57}\right)}{1+\exp \left(\frac{4(100-0)}{\sqrt{2 \pi} * 5055.57}\right)}-\frac{\exp \left(\frac{4(-100-0)}{\sqrt{2 \pi} * 5055.57}\right)}{1+\exp \left(\frac{4(-100-0)}{\sqrt{2 \pi} * 5055.57}\right)}
$$

$$
\begin{aligned}
& P_{t}=\frac{\exp \left(\frac{4(350-10817.13)}{\sqrt{2 \pi} * 4928.08}\right)}{1+\exp \left(\frac{4(350-10817.13)}{\sqrt{2 \pi} * 4928.08}\right)}-\frac{\exp \left(\frac{4(-350-10817.13)}{\sqrt{2 \pi} * 4298.02}\right)}{1+\exp \left(\frac{4(-350-10817.13)}{\sqrt{2 \pi} * 4928.08}\right)} \\
& P_{r}=0.3044095 \text { and } P_{t}=0.006438
\end{aligned}
$$

The total collision probability is obtained by multiplying the $P_{r}$ and $P_{t}$.

$$
\mathrm{P}=1.960 * 10^{-3}
$$

The red threshold collision probability is the collision probability which are there are great chances of collision. The red collision probability defined by ESA is in the range of $10^{-4}$. The obtained collision probability obtained clearly shows that the assurance of collision which can be visualized in the software.

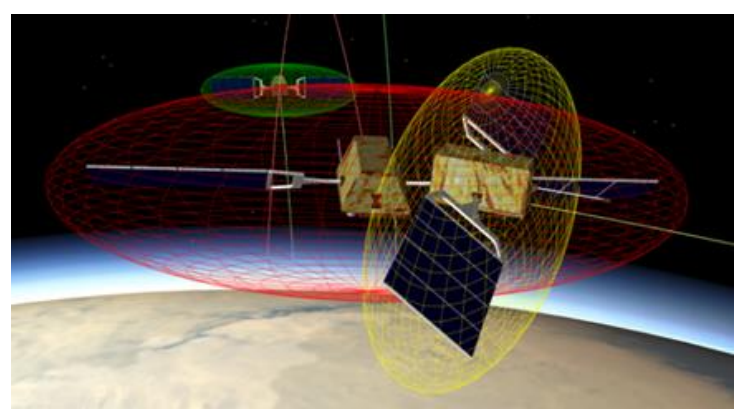

Fig. 3. Collision of Iridium 73 and Taurus

B. Validation of the collision probability

Lie Chen et.al, [2] derived the explicit expression for calculating collision probability assuming the orbit are circular or near circular orbit. They considered US and Russian satellite collision event and the state vectors are given in Table 4.

This initial state vector is applied in the FreeFlyer and it was propagated for the couple of hours and the position and velocity vectors were taken. The standard deviation was founded. The collision probabilities were calculated using previous calculation.

The angle between velocity vectors and their magnitude ratio are found to be

$$
\eta=\frac{7.46677}{7.47217}=0.99 \text { and } \varphi=\cos ^{-1} \frac{v 1 . v 2}{V 1 * V 2}=102.458 \text { degree }
$$

TABLE 4: INITIAL STATE VECTOR OF THE TWO SATELLITES

\begin{tabular}{|c|c|c|c|c|c|c|c|}
\hline Satellite & $\mathbf{X}(\mathbf{k m})$ & $\mathbf{Y}(\mathbf{k m})$ & $\mathbf{Z}(\mathbf{k m})$ & $\mathbf{V x}(\mathbf{k m} / \mathbf{s})$ & $\mathbf{V y}(\mathbf{k m} / \mathbf{s})$ & $\mathbf{V z}(\mathbf{k m} / \mathbf{s})$ & $\mathbf{E c c e n t r i c i t y}$ \\
\hline US satellite & -1457.27 & 1589.56 & 6814.18 & -7.00 & -2.43 & -0.926 & 0.001174 \\
\hline Russian Satellite & -1457.53 & 1588.9 & 6814.31 & 3.57 & -6.17 & 2.20 & 0.000660 \\
\hline
\end{tabular}

The standard deviation in radial and transverses direction are found to be:

$$
\sigma_{r}==4356.886 \mathrm{~km} \text { and } \sigma_{t}=6387.9253 \mathrm{~km}
$$

The altitude difference and closet approach distance was found to be:

$$
\Delta \mathrm{h}=10.277 \mathrm{~km}
$$

$$
\rho_{\min }=10713.123
$$

The collision probability is obtained by substituting the known values in Eq. (10) and is compared with the existing explicit collision probability result [2] is tabulated in table 5. 
TABLE 5: VALIDATION OF COLLISION PROBABILITY

\begin{tabular}{|c|c|c|}
\hline $\begin{array}{l}\text { The explicit collision } \\
\text { probability }\end{array}$ & $\begin{array}{l}\text { The calculated } \\
\text { collision probability }\end{array}$ & Error \\
\hline $1.807975 \times 10^{-4}$ & $1.9000 \times 10^{-4}$ & 0.050899 \\
\hline
\end{tabular}

C. The collision avoidance maneuver

The collision avoidance maneuver is essential to safeguard the collision between satellites by performing impulsive burn which is made to avoid the contact between the primary and secondary satellites. Here, the Iridium 73 is considered as primary satellite and Taurus, the dead satellite is considered equivalent to debris. The impulsive burn is applied only to the primary satellite, that is, Iridium 73. The range of altitudes chosen for maneuvering the satellite was within $7100-7500 \mathrm{~km}$ of semi-major axis variation. The other satellite within this region was plotted in FreeFlyer. The maneuver was done using trial and error method and the outcomes are tabulated in table 6.

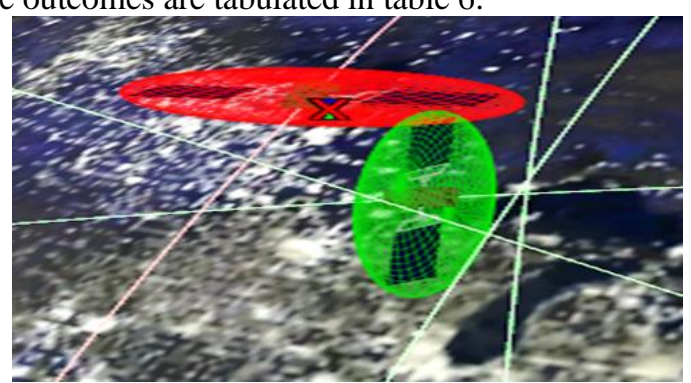

Fig. 4(a). Collision with OV21 at $\Delta \mathrm{V}$ of $0.09 \mathrm{~km} / \mathrm{s}$ at TA $220^{\circ}-240^{\circ}$

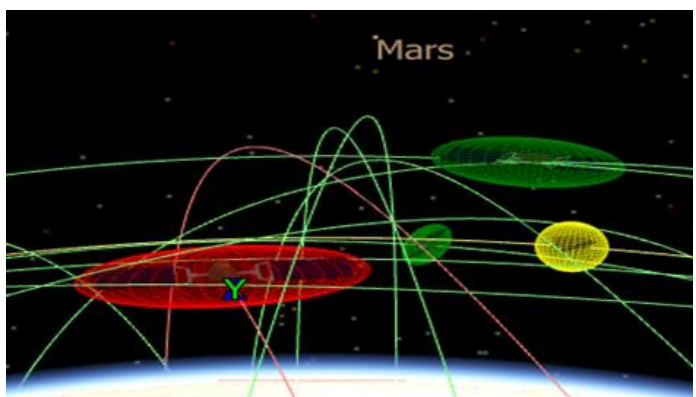

Fig. 4(b). Iridium 73 and Taurus maneuvered safely at $\Delta \mathrm{V}$ of $0.1 \mathrm{~km} / \mathrm{s}$ at $\mathrm{TA}$ $220^{\circ}-240^{\circ}$

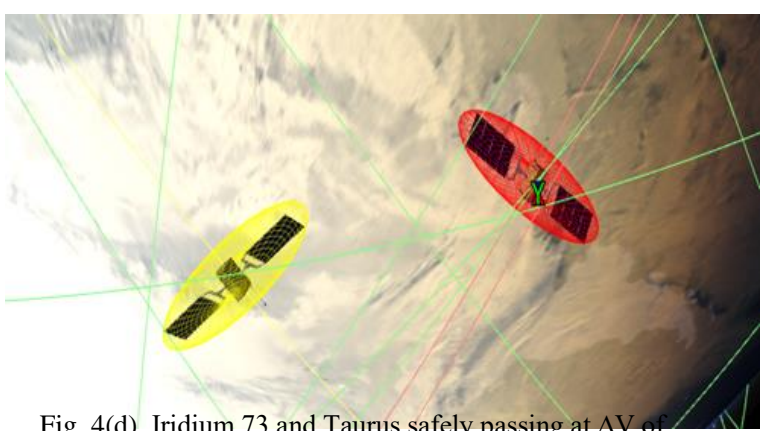

Fig. 4(d). Iridium 73 and Taurus safely passing at $\Delta V$ of $0.1 \mathrm{~km} / \mathrm{s}$ at TA $240^{\circ}-260^{\circ}$

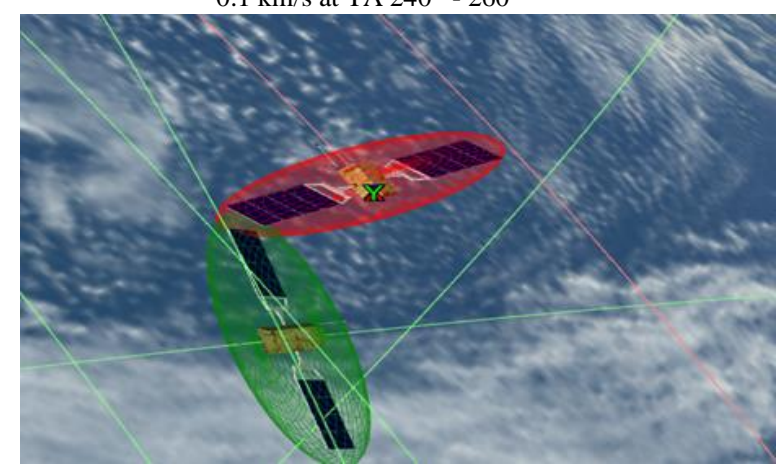

Fig. 4(e). Iridium 73 collision with transient $2 \mathrm{~A}$ burn at TA $240^{\circ}-260^{\circ}$

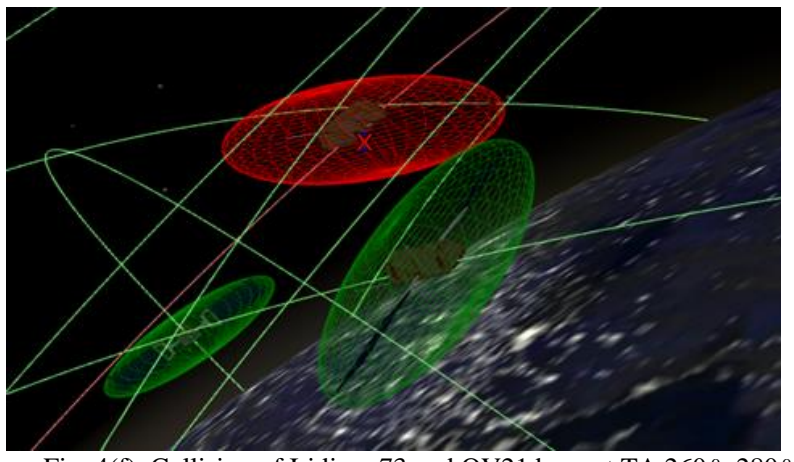

Fig. 4(f). Collision of Iridium 73 and OV21 burn at TA $260^{\circ}-280^{\circ}$

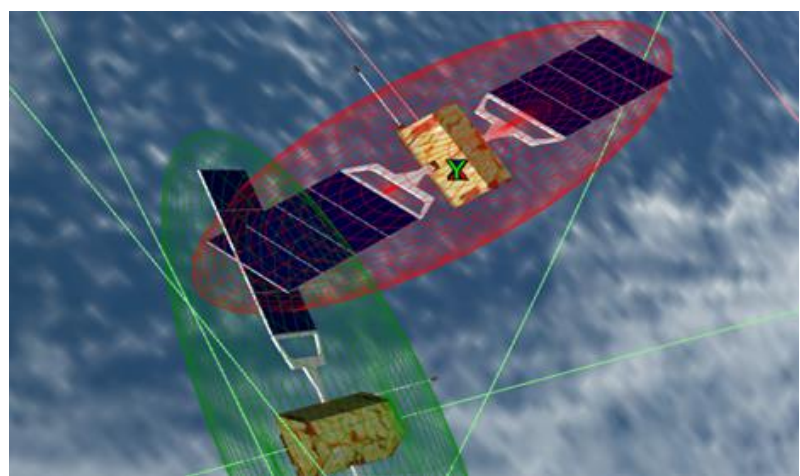

Fig. 4(g). Collision of Iridium 73 and OV21 burn at TA $280^{\circ}-300^{\circ}$

TABLE 6: OUTCOMES OBTAINED USING TRIAL AND ERROR MANEUVER IN FREEFLYER.

\begin{tabular}{|c|c|c|c|}
\hline $\begin{array}{c}\text { Delta-V }(\Delta \mathbf{V}) \\
(\mathbf{k m} / \mathbf{s})\end{array}$ & $\begin{array}{c}\text { True anomaly } \\
(\mathbf{T A}) \text { (Degree) }\end{array}$ & Altitude $(\mathbf{k m})$ & Outcome \\
\hline 0.09 & $220-240$ & 7277 & Collision with Ov21 \\
\hline 0.1 & $220-240$ & 7297.8 & Safe \\
\hline 0.1 & $240-260$ & 7298.0 & Collision with transient 2a \\
\hline 0.1 & $260-280$ & 7298.33 & Collision with transient2A \\
\hline 0.1 & $280-300$ & 7298.66 & $\begin{array}{c}\text { Collision with Ov21 and Transient } \\
\text { 2A }\end{array}$ \\
\hline 0.1 & $300-320$ & - & Collision with Taurus \\
\hline
\end{tabular}




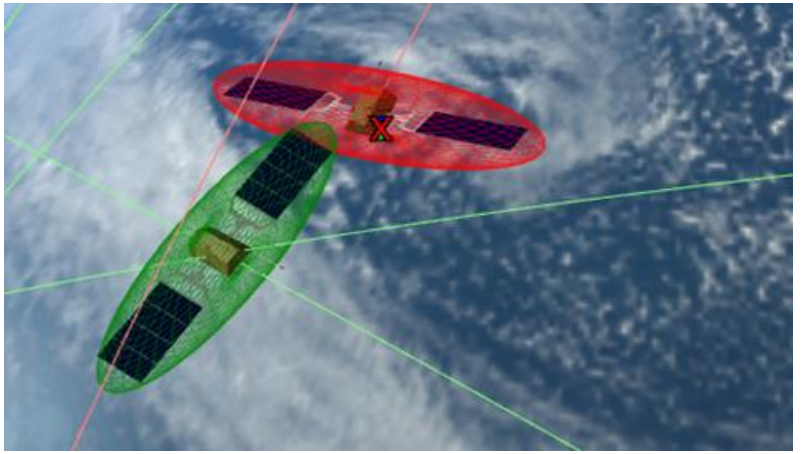

Fig. 4(h). Collision of Iridium 73 and transient 2 a burn at TA $280^{\circ}-300^{\circ}$

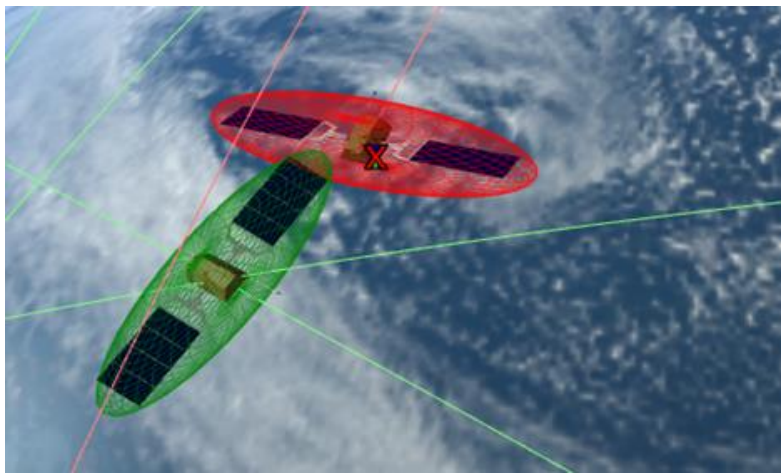

Fig. 4(i). Collision of Iridium 73 and Taurus burn at TA $300^{\circ}-320^{\circ}$

D. Two-point mass case and $\mathbf{J} 2$ perturbed

The primary satellite was maneuvered under point mass force model and the only force acting on the satellite will be the gravitational pull of the earth, assumed to be a perfect sphere and mass concentrated at its center. The satellite was then maneuvered by varying the delta- $\mathrm{V}$, which is impulsive burn over a different range of true anomalies and the optimum delta$\mathrm{V}$ was found in order to safely perform the maneuver.

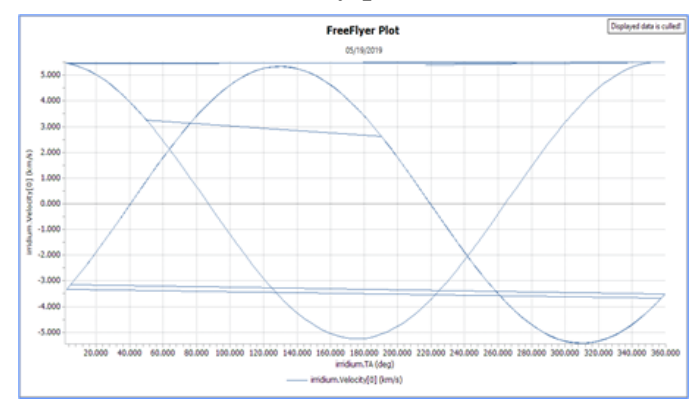

Fig 5(a). Plot between Iridium TA and Velocity magnitude

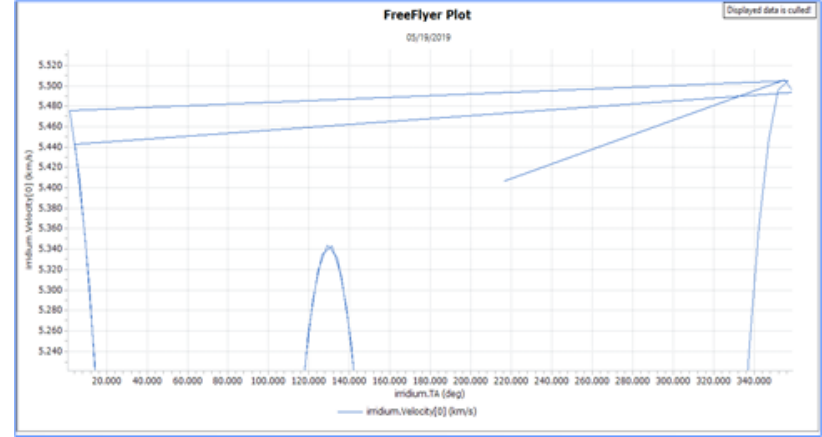

Fig 5(a). (b)Plot shows the increase in velocity due to burn

The decrease in the velocity can been seen in Fig.5(a), because of the negative impulsive burn given in-order to bring the satellite to its original orbit. Fig. 5(b) shows the increase in the velocity due to impulsive burn.

The optimum delta- $\mathrm{V}$ is $0.1 \mathrm{~km} / \mathrm{s}$ at true anomaly of $220-240$ degree. The $\mathrm{J} 2$ perturbation was applied to this true anomaly and found the required delta- $\mathrm{V}$ for safe maneuvering of the satellite. (Table 7).

The method to calculate collision probability between two near-circular satellite were calculated. The MATLAB program was developed to convert the two-line element into the state vectors. The satellite was programmed into FreeFlyer and propagated in order to protect primary satellite. The minimum delta- $\mathrm{V}$ was found to be $0.1 \mathrm{~km} / \mathrm{s}$ for a two-point mass force model. The minimum delta-V was found to be $0.083 \mathrm{~km} / \mathrm{s}$ for the $\mathrm{J} 2$ perturbed force model. The relation between the final mass of ideal and $\mathrm{J} 2$ component was derived.

The delta- $\mathrm{V}$ obtained under the $\mathrm{J} 2$ perturbated case is slightly lesser than the two-point mass, this deviation is due to the effect of additional mass concentration near the equator than the pole region of the earth's surface. The additional gravitational pull in the equator provides a slight slingshot effect, which gives the additional momentum to the satellite. This additional momentum provides a higher velocity of the satellite providing lesser delta$\mathrm{V}$. Hence, the mass of propellant carried will be lesser in the $\mathrm{J} 2$ force model case than the mass of propellant carried in the twopoint mass case.

\section{E. Relation between $\mathrm{M}_{2 \mathrm{j}}$ and $\mathrm{M}_{1 \mathrm{j}}$}

The delta- $\mathrm{V}$ for both ideal and $\mathrm{J} 2$ perturbed cases are known. The rocket equation can be used to obtain the relation between $M_{2 j}$ and $M_{1 j}$.

TABLE 7: EFFECT OF J2 PERTURBATION ON DELTA-V.

\begin{tabular}{|c|c|c|}
\hline Parameter & Two-point mass & J2-perturbated \\
\hline Delta- V & $0.1 \mathrm{~km} / \mathrm{s}$ & $0.083 \mathrm{~km} / \mathrm{s}$ \\
\hline
\end{tabular}

The rocket equation is given by

$$
\Delta V=I_{s p} * g * \ln \frac{M_{1}}{M_{2}}
$$

where, $\Delta \mathrm{V}$ is required change in velocity for maneuvering the satellite from one orbit to another. Isp is a specific impulse which defines the property of the propellant used and $\mathrm{g}$ is the acceleration due to gravity. M_1 is the launch mass of the entire system. M_2 is the final mass of the satellite in the orbit.

Delta- $\mathrm{V}$ for unperturbed maneuver

$$
0.1=I_{s p} * g * \ln \frac{M_{1}}{M_{2 i}}
$$


Delta-V for perturbed maneuver

$$
0.083=I_{s p} * g * \ln \frac{M_{1}}{M_{2 j}}
$$

where, $M_{2 i}$ is the final mass of the satellite in two-point mass case (without perturbation) and $M_{2 j}$ is final mass of the satellite in the J2 perturbated case.

Dividing both the equations:

$$
1.2=\frac{\ln \frac{M_{1}}{M_{2 i}}}{\ln \frac{M_{1}}{M_{2 j}}}
$$

By re-arranging the terms

$$
M_{2 i}=\frac{M_{2 j}{ }^{1.2}}{M_{1}{ }^{0.2}}
$$

The relation shows the final mass of the satellite relating ideal and J2 perturbed case. The final mass signifies the amount of propellant required to be carried.

\section{CONCLUSION}

The collision probability between Iridium and Taurus satellites were calculated by considering only the two-line element data as an initial value. The Iridium satellite was considered as a primary satellite and the Taurus satellite was the secondary satellite. The Taurus satellite was generalized to a dead satellite that could be debris or any other enemy satellite. The Taurus was uncontrollable throughout the maneuvering sequence. The impulsive burn was only given to the Iridium satellite.

The impulsive burn was performed to the Iridium satellite under two-cases. The two-point mass case where no perturbating cases were considered. The $\mathrm{J} 2$ perturbated case considers the effect of the earth's oblateness on the account. The delta- $\mathrm{V}$ required to successfully perform collision avoidance maneuver under $\mathbf{J} 2$ perturbed case is lesser than the delta- $\mathrm{V}$ required under a two-point case. The relation between the final mass of the satellite in orbit for the point-mass case and $\mathrm{J} 2$ perturbated case was derived. The relation shows that the mass of propellant required to perform collision avoidance maneuver under the $\mathbf{J} 2$ force model is less than the mass of propellant required to perform collision avoidance maneuver under a twopoint mass force model.

\section{REFERENCES}

[1] Patera, R. 2001. "General Method for Calculating Satellite Collision Probability". Journal of Guidance, Control, and Dynamics, 24(4), pp. 716-722.

[2] Chen, Lei \& Bai, Xian-Zong \& Liang, Yan-Gang \& Li, Kebo. 2017. "Orbital Data Applications for Space Objects". 10.1007/978-981-102963-9.

[3] Xu, X. and Xiong, Y. 2014. "A method for calculating probability of collision between space objects". Research in Astronomy and Astrophysics, 14(5), pp.601-609.

[4] Bombardelli, Claudio \& Hernando-Ayuso, Javier \& Pelayo, R.G. 2014. "Collision avoidance maneuver optimization". Advances in the Astronautical Sciences. 152. 1857-1870

[5] CelesTrak.com. 2020. CelesTrak: Current NORAD Two-Line Element Sets. [online] Available at: https://www.celestrak.com/NORAD/elements/.

[6] CelesTrak.com. 2020. CelesTrak: SOCRATES. [online] Available at: https://www.celestrak.com/SOCRATES/

[7] Curtis, H. 2013. "Orbital mechanics for engineering students". 3rd ed Butterworth-Heinemann.

[8] Bate, R., Mueller, D., White, J. and Saylor, W. 1971. "Fundamentals of astrodynamics". 1st ed. New York: Dover Publications.

[9] Danielson, D., Latta, G., Sagovac, C., Krambeck, S. and Snider, J. 1993. "Satellite motion around an oblate planet". Monterey, Calif. Naval Postgraduate School.

[10] Esa.int. 2020. Debris objects in low-Earth orbit (LEO). [online] Available at: http://www.esa.int/ESA_Multimedia/Images/2008/03/Debris_objects in_low-Earth_orbit_LEO2

[11] Geography.hunter.cuny.edu. 2005. The earth's shape is a spheroid. [online] Available at: http://www.geography.hunter.cuny.edu/ jochen/GTECH361/lectures /lecture04/concepts/Datums/The\%20earths\%20shape\%20is\%20a\%2 0spheroid.htm.

[12] Wikiwand. 2020. Space debris | Wikiwand. [online] Available at: https://www.wikiwand.com/en/Space_debris. 